\title{
Análise exergética de um sistema de evaporação de múltiplos estágios para a concentração de licor negro
}

\author{
Exergy analysis of a multistage evaporation system for black liquor concentration \\ Análisis de exergía de un sistema de evaporación multietapa para la concentración de licor negro
}

Recebido: 04/11/2021 | Revisado: 12/11/2021 | Aceito: 15/11/2021 | Publicado: 24/11/2021

Lucas Nunes Rodrigues

ORCID: https://orcid.org/0000-0003-2412-836X Instituto Federal de Educação Ciência e Tecnologia do Piauí, Brasil E-mail: lucas-rodrigues07@outlook.com

Luiz Felipe Rodrigues Silva

ORCID: https://orcid.org/0000-0002-0384-815X Instituto Federal de Educação Ciência e Tecnologia do Piauí, Brasil

E-mail: luizfeliperods1@gmail.com

Matheus de Sousa Borges

ORCID: https://orcid.org/0000-0002-5611-2197 Instituto Federal de Educação Ciência e Tecnologia do Piauí, Brasil E-mail: matheus.dsb151099@gmail.com

Karine Matos Sousa

ORCID: https://orcid.org/0000-0003-0221-2368 Instituto Federal de Educação Ciência e Tecnologia do Piauí, Brasil

E-mail: karine.sous@hotmail.com

Francisco Valdivino Rocha Lima

ORCID: https://orcid.org/0000-0002-4827-2545 Instituto Federal de Educação Ciência e Tecnologia do Piauí, Brasil E-mail: valdivino@ifpi.edu.br

Anderson Felipe Chaves Fortes

ORCID: https://orcid.org/0000-0002-6190-1035 Instituto Federal de Educação Ciência e Tecnologia do Piauí, Brasil E-mail: anderson.fortesem@ifpi.edu.br

\begin{abstract}
Resumo
As indústrias de papel e celulose, no Brasil, são grandes consumidoras de energia, dispondo de uma região ideal para o desenvolvimento de florestas do eucalipto. O processo Kraft é o mais empregado no país por ser capaz de reutilizar os produtos químicos, sendo o licor negro um dos subprodutos, substância complexa com composição orgânica e inorgânica. Para obter viabilidade econômica e ambiental, a parcela inorgânica deve ser readquirida em uma planta de recuperação para ser reutilizada, com a planta dispondo de um sistema de evaporação de múltiplos estágios, responsável por concentrar o licor para ser queimado na caldeira de recuperação. Em face do exposto, o objetivo desse trabalho foi o desenvolvimento e validação de um modelo matemático fenomenológico que representasse o sistema de evaporação de múltiplos estágios, para realizar um estudo de temperatura e pressão de entrada que trouxessem os melhores resultados de economia de vapor, eficiência exergética, destruição de exergia e de teor de sólidos do licor negro. Dessa forma, foram encontrados as melhores condições de entrada e os evaporadores que detêm maiores taxas de destruição de exergia. Para isso, o modelo foi construído baseado nos trabalhos de pesquisa afim, utilizando balanços de massa, energia e equilíbrio de fases, assim como retratou satisfatoriamente os resultados encontrados pelo autor de referência, obtendo-se o maior desvio de $0,2 \%$. Este trabalho possibilitou um avanço na compreensão dos fenômenos envolvidos e a otimização desse processo, visando uma melhoria no consumo energético do meio.
\end{abstract}

Palavras-chave: Licor negro; Evaporação; Exergia; Termodinâmica; Eficiência.

\begin{abstract}
The pulp and paper industries in Brazil are large consumers of energy, offering an ideal region for the development of eucalyptus forests. The Kraft process is the most used in the country as it is capable of reusing chemical products, with black liquor being one of the by-products, a complex substance with organic and inorganic composition. To obtain economic and environmental viability, the inorganic portion must be reacquired in a recovery plant to be reused, with the plant having a multi-stage evaporation system, responsible for concentrating the liquor to be burned in the recovery boiler. In light of the above, the objective of this work was the development and validation of a phenomenological mathematical model that would represent the multistage evaporation system, to carry out a study of inlet temperature and pressure that would bring the best results in terms of steam savings, exergetic efficiency, exergy destruction and
\end{abstract}


black liquor solids content. Thus, the best inlet conditions and evaporators that have higher exergy destruction rates were found. For this, the model was built based on related research works, using mass, energy and phase balance balances, as well as satisfactorily portraying the results found by the reference author, obtaining the largest deviation of $0.2 \%$. This work allowed an advance in the understanding of the phenomena involved and the optimization of this process, aiming at an improvement in the energy consumption of the environment.

Keywords: Black liquor; Evaporation; Exergy; Thermodynamics; Efficiency.

\section{Resumen}

Las industrias de celulosa y papel en Brasil son grandes consumidoras de energía, ofreciendo una región ideal para el desarrollo de los bosques de eucaliptos. El proceso Kraft es el muy utilizado en el país ya que es capaz de reutilizar productos químicos, siendo uno de los subproductos el licor negro, sustancia compleja de composición orgánica e inorgánica. Para obtener viabilidad económica y ambiental, la porción inorgánica debe ser readquirida en una planta de recuperación para ser reutilizada, disponiendo la planta de un sistema de evaporación multietapa, responsable de concentrar el licor a quemar en la caldera de recuperación. A la luz de lo anterior, el objetivo de este trabajo fue el desarrollo y validación de un modelo matemático fenomenológico que representaría el sistema de evaporación multietapa, para realizar un estudio de temperatura y presión de entrada que arrojara los mejores resultados en términos de ahorro de vapor, eficiencia, exergética, destrucción de exergía y contenido de sólidos de licor negro. Por lo tanto, se encontraron las mejores condiciones de entrada y evaporadores que tienen mayores tasas de destrucción de exergía. Para ello, el modelo se construyó a partir de trabajos de investigación relacionados, utilizando balances de masa, energía y balance de fases, además de retratar satisfactoriamente los resultados encontrados por el autor de referencia, obteniendo la mayor desviación del 0,2\%. Este trabajo permitió avanzar en la comprensión de los fenómenos involucrados y la optimización de este proceso, con el objetivo de mejorar el consumo energético del medio ambiente. Palabras clave: Licor negro; Evaporación; Exergía; Termodinámica; Eficiencia.

\section{Introdução}

Os evaporadores de múltiplos estágios são largamente utilizados na indústria, sendo destaque sua aplicação na produção sucroalcooleira, de leite em pó, café, celulose e na dessalinização da água (Bhargava, 2008). Com o avanço dos meios eletrônicos de comunicação, a partir da década de 80, o uso do papel deteve crescimento significativo. Esse consumo poderá ter um aumento de 1,6\% a.a. até 2025 (Silva et al., 2016). Além disso, as exportações de papel no Brasil cresceram em 10,7\% no ano de 2018 (Ibá, 2019; BNDES, 2010).

O processo Kraft é o mais usado no país para a produção de papel, pois o diferencial dele está no ciclo de restauração de produtos químicos, que viabiliza de forma econômica e ambiental (Andreuccetti, 2019; Ferreira, 2013). Um dos subprodutos desse processo é uma solução complexa, formada por substâncias orgânicas e inorgânicas, chamada licor negro (Carreiro, 2009; Batista, 2018). Neste processo, as fibras de celulose são separadas da lignina, por processos reacionais. Estas reações ocorrem em um digestor pressurizado, onde a madeira é aquecida com licor de cozimento, composto de hidróxido de sódio ( $\mathrm{NaOH}$ ) e sulfeto de sódio (Na2S). Os produtos da digestão são a polpa de celulose e o licor negro (Passini, 2017; Campos, 2009).

As indústrias de papel e celulose são grandes consumidoras de energia (Diel, 2013). A planta de evaporação, constituída por um sistema de evaporação de múltiplos efeitos, consome parte significativa dessa energia. Em muitos casos, a planta de evaporação representa 30-35\% da demanda energética total do processo de fabricação (Olsson, 2009).

A fim de verificar a relevância do presente tema no meio acadêmico, realizou-se uma revisão da literatura. Bhargava et al. (2008), por exemplo, desenvolveu um modelo matemático no processo de simulação do evaporador de efeito múltiplo, selecionado para a investigação de um sistema evaporador de filme plano queda de efeito sétuplo. Com as condições adotadas aplicadas aos cálculos virtuais, encontrou-se ótima eficiência com sistema de evaporação de sete efeitos. Em paralelo, Diel (2013) usou o método de Newton para validar a matemática da pesquisa anterior. O autor, valendo-se ainda dos evaporadores de 7 efeitos, objetivou em sua tese a economia, com perdas de calor desprezíveis. Somando-se aos demais, Carreiro (2009) realizou uma análise experimental sobre a eficiência exergética de uma indústria brasileira produtora de papel e celulose. Por meio dessas pesquisas, constatou-se, além da importância, lacunas a serem estudadas acerca da temática.

Nesse contexto, o objetivo deste artigo é uma simulação e análise exergética do sistema de evaporação de múltiplos 
efeitos, para identificar quais condições de entrada que resultassem em uma melhor eficiência exergética do sistema, na taxa de concentração do licor negro, além disso, identificar os volumes de controle com maiores taxas de destruição de exergia.

Para atingir os objetivos propostos, o primeiro passo dessa pesquisa foi a discretização das equações, para as devidas análises, a partir dos trabalhos tomados como referência bibliográfica. Em seguida, desenvolveu-se a solução, por meio de ferramentas computacionais, como o Excel e EES. Por fim, parametrizou-se as variáveis de entrada, como vazão mássica de entrada e pressão no primeiro módulo, de maneira que se possa aumentar, de modo geral, a eficiência exergética do ciclo a ser avaliado. $\mathrm{O}$ trabalho está estruturado da seguinte forma: além desta introdução, na seção 2 é exposta a metodologia; os resultados e discussão encontram-se na seção 3; e na seção 4 é apresentada a conclusão.

\section{Metodologia}

A metodologia empregada nessa pesquisa consiste em duas etapas: pesquisa bibliográfica e equacionamento dos fenômenos envolvidos no processo. Durante as etapas, busca-se entender os comportamentos dos fenômenos do ciclo, as condições técnicas de funcionamento e parametrização das variáveis de entrada, para que se possa encontrar, de modo geral, a melhor eficiência do sistema. Abaixo segue a descrição de cada etapa.

\subsection{Pesquisa bibliográfica}

As fontes de pesquisas que serão utilizadas no trabalho são: livros, artigos, trabalhos de conclusão de curso, dissertações, teses, normas técnicas, entre outros, sendo que alguns deles já estão listados nesse projeto. Quatro bases de dados foram selecionadas: Science Direct; Scopus; Web of Science; e SciELO. A escolha dessas bases justifica-se pela amplitude, qualidade dos periódicos indexados e suas funções de busca. Cada base de dados será consultada de acordo com a seguintes strings de busca: "Evaporadores AND Exergia" OR "Evaporadores AND Liquor” OR "Evaporadores AND Efficiency".

\subsection{Estudo e equacionamento dos fenômenos envolvidos no processo}

O sistema de evaporadores de múltiplos efeitos consiste em um número de evaporadores em série com a possibilidade de possuir evaporadores em paralelo (Stape, 2017; Marcelino, 2019). A fonte externa de calor poder ser vapor ou água quente, atuando no primeiro efeito (evaporador) fazendo com que a água presente na substância a ser concentrada dentro do efeito evapore. Esse vapor gerado atua como fonte de calor para o segundo efeito, onde a água não evaporada do primeiro efeito é direcionada. Esse processo se repete até o último efeito (Moreira, 2018; Mendes, 2021). Com o intuito de gerar viabilidade de execução desse trabalho, o sistema de evaporação será modelado com sete efeitos, os evaporadores serão considerados como adiabáticos, perdas de calor e pressão nos tubos, que interligam os evaporadores. Serão desconsideradas, a vazão de vapor vivo, no início do sistema, e o fluxo de energia usada, para o bombeamento do licor, serão considerados como constantes, as condições de estado morto serão consideradas de $27^{\circ} \mathrm{C}$ e $100 \mathrm{kPa}$. Abaixo, na Figura 1, é ilustrado o sistema estudado: 


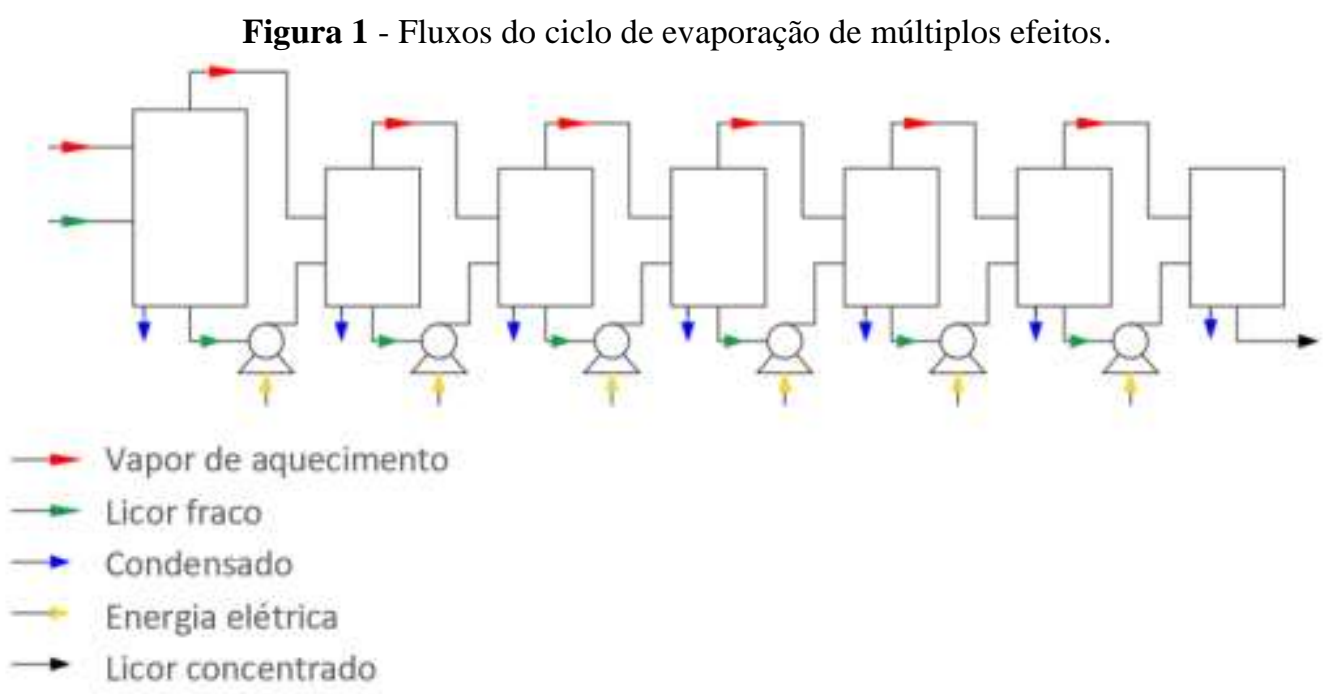

Fonte: Elaboração própria (2021).

Além disso, para realizar as simulações e análises do sistema de evaporação de múltiplos efeitos e alcançar os objetivos propostos, os comportamentos físicos, químicos, termodinâmicos, conservação de massa do licor negro e do vapor de água foram modelados de acordo com equações encontradas na literatura.

\subsection{Discretização das equações das propriedades do licor}

Para Seleghim (2015), o teor de sólidos pode ser obtido com a equação 1, onde o teor de sólidos de saída é igual a vazão mássica de sólidos, dividido pela diferença da vazão do líquido com a do vapor na mistura, somada com a vazão mássica de sólidos.

$$
C_{\text {brix }}=\frac{\dot{m}_{\text {sólidos }}}{\dot{m}_{\text {sólidos }}+\left(\dot{m}_{l i q}-\dot{m}_{\text {vap }}\right)}
$$

No ciclo de múltiplos estágios, a partir de cada efeito, ocorre uma perda de pressão e temperatura, podendo esta última ser calculada através da equação 2 , onde, segundo o autor, está diretamente ligada à concentração de sólidos, referente ao efeito em questão.

$$
\Delta T_{\text {ebulição }}=\frac{2 C_{\text {brix }}}{\left(1-C_{\text {brix }}\right)}
$$

Ainda em concordância com Seleghim (2015), o cálculo do fluxo do balanço de massa é obtido a partir das equações 3 e 4, que estão ligadas às entalpias e vazões mássicas. Na equação 3, considera-se o primeiro caso como uma expansão isoentálpica, onde parte do vapor é gerado pelo "flash amento", por meio da válvula de expansão, enquanto o segundo caso corresponde ao restante do vapor gerado, através da sua condensação para o estado seguinte.

$$
\dot{m}_{v, k}=\dot{m}_{l, k-1}\left(\frac{h_{l, k-1}-h_{l, k}}{h_{v, k}-h_{l, k}}\right)_{\text {caso } 1}+\dot{m}_{v, k-1}\left(\frac{h_{v, k-1}-h_{l, k-1}}{h_{v, k}-h_{l, k}}\right)_{\text {Caso } 2}
$$

A equação 4 está ligada ao diagrama PH, em que a vazão da mistura do efeito é a diferença entre a vazão do líquido no efeito anterior com a do vapor gerado a partir dele.

$$
\dot{m}_{l, k}=\dot{m}_{l, k-1}-\dot{m}_{v, k}
$$


As equações, para o cálculo da entalpia e entropia do licor negro, são obtidas a partir das equações 5 e 6 (Carreiro, 2009).

$$
\begin{aligned}
& h=h_{0}+\int_{T_{0}}^{T} C_{P}(T) d T \\
& s=s_{0}+\int_{T_{0}}^{T} \frac{C_{P}(T)}{T} d T
\end{aligned}
$$

Sendo $h$ e $s$ a entalpia e a entropia, respectivamente, de cada elemento químico do licor e o subscrito 0 o estado de referência (Estado Morto). A soma dos valores encontrados, para cada substância, será a entalpia e entropia do licor, como apontado por Carreiro (2009).

\subsection{Discretização das equações de energia e exergia}

A equação da primeira lei da termodinâmica ou equação do balanço de energia aplicada a um volume de controle com escoamento em regime permanente é dada pela equação 7, (Çengel \& Boles, 2013).

$$
\dot{Q}-\dot{W}=\sum_{s a i} \dot{m}\left(h+\frac{v^{2}}{2}+g z\right)
$$

Sendo $\dot{Q}$ o fluxo de calor, $\dot{W}$ o trabalho, mo fluxo mássico, h a entalpia do fluido, v a velocidade do escoamento, g a gravidade e $\mathrm{z}$ a altura em relação ao nível de referência nulo.

Também em Çengel e Boles (2013), a exergia de escoamento ( $\psi$ ) é dada pela equação 8, sendo h a entalpia, T a temperatura, $\mathrm{S}$ a entropia e o subscrito 0 o estado de referência.

$$
\psi=\left(h-h_{0}\right)+T_{0}\left(S-S_{0}\right)
$$

Na visão de Kotas (1985), a exergia é o máximo trabalho disponível que pode ser obtido de uma determinada forma de energia utilizando as condições da atmosfera como referencial dos estados. O balanço de exergia, para escoamento em regime permanente, é dado pela equação 9 , (Çengel \& Boles, 2013). Sendo o subscrito k utilizado para indicar o estado e $\dot{X}_{\text {destruída }}$ a exergia destruída no processo analisado.

$$
\sum\left(1-\frac{T_{0}}{T_{K}}\right) \dot{Q}_{K}-\dot{W}+\sum \dot{m} \varphi_{\text {ent }}-\sum \dot{m} \varphi_{s a i}-\dot{X}_{\text {destrída }}=0
$$

A eficiência de segunda lei é dada pela equação 10, (Çengel e Boles, 2013). Sendo $\dot{X}_{\text {fornecida }}$ a diferença entre a exergia fornecida e destruída no efeito anterior.

$$
\eta_{I I}=1-\frac{\dot{X}_{\text {destruída }}}{\dot{X}_{\text {fornecida }}}
$$

Em conformidade com Carreiro (2009), a exergia dos sólidos é dada pela equação 11. Sendo $n_{m s}$ a massa de cada componente químico, $b_{M S}^{o}$ a exergia química padrão e $n_{k}$ a concentração de cada elemento químico. 


$$
B_{S}=n_{m_{s}} \times b_{M_{S}}^{\circ}+\sum n_{k} \times\left[\left(h-h_{0}\right)-T_{0}\left(s-s_{0}\right)\right]
$$

Segundo Gallo (1998) a eficiência exergética é a relação entre a variação da exergia desejada e o consumo de exergia necessária ao processo. Conforme Carreiro (2009), a eficiência exergética consiste na relação entre a variação da exergia desejada e a exergia necessária ao processo, como mostrado na equação 12.

$$
\Psi=\frac{\text { Exergia do LP concentrado-Exergia do LP } 15 \%}{\text { Exergia vapor vivo-(Exergia condensado }+ \text { Exergia condensado evaporado })+E E}
$$

Para a análise de todo o processo descrito acima, podemos observar que além da eficiência energética também foi considerado a eficiência exergética do sistema, que por sua vez é utilizada para avaliar o desempenho do sistema baseado na segunda lei da termodinâmica. Portanto, segundo Cao et al. (2017), temos um melhor indicador na análise exergética em comparação a energética, pois tanto a quantidade quanto a qualidade da energia são consideradas.

\section{Resultados e Discussão}

Como apresentado, foi adotada uma faixa de valores de pressões para analisar qual possui a melhor eficiência exergética. Como efeito de validação do modelo matemático, foi usado, inicialmente, o valor de $400 \mathrm{kPa}$, em comparação com os resultados encontrados por Carreiro (2009). A Tabela 1 a seguir ilustra os resultados encontrados.

Tabela 1 - Comparação de resultados com a literatura de referência.

\begin{tabular}{|c|c|c|}
\hline Parâmetros comparados & Eficiência exergética (\%) & Teor de sólidos (\%) \\
\hline Carrero & 42,40 & 72,00 \\
\hline Modelo & 42,20 & 72,19 \\
\hline Desvio & 0,20 & 0,19 \\
\hline
\end{tabular}

Fonte: Elaboração própria (2021).

O modelo reproduziu bem os resultados do trabalho realizado por Carreiro (2009), sendo o maior desvio percentual de 0,20\% . Dessa forma, o parâmetro foi validado, podendo predizer com uma boa precisão os resultados do autor tomado como referência, garantindo confiabilidade nos próximos resultados encontrados pelo mesmo.

Sabendo-se que o modelo representou os resultados de Carreiro (2009) com grande proximidade, a faixa de valores de pressões, citada anteriormente, foi aplicada ao modelo para calcular a Eficiência de Segunda Lei em cada caso, teor de sólidos no final do processo e a porcentagem de destruição de exergia. Esses resultados foram ilustrados na Tabela 2 a seguir.

Tabela 2 - Resultados de eficiência de Segunda Lei, teor de sólidos e destruição de exergia a partir de faixa de valores de pressão.

\begin{tabular}{|c|c|c|c|}
\hline Pressão de entrada (kPa) & $\begin{array}{c}\text { Eficiência de } \\
\text { segunda lei (\%) }\end{array}$ & Teor de Sólidos (\%) & $\dot{X}$ destruída (\%) \\
\hline 380 & 45,86 & 74,38 & 51,32 \\
\hline 390 & 44,02 & 73,17 & 52,89 \\
\hline 400 & 42,20 & 72,07 & 50,99 \\
\hline 410 & 40,73 & 71,01 & 55,59 \\
\hline 420 & 39,26 & 70,04 & 58,81 \\
\hline
\end{tabular}

Fonte: Elaboração própria (2021). 
A partir desses, observou-se que há uma diminuição não só da Eficiência de Segunda Lei como também no teor de sólidos, no final do processo de evaporação, com o aumento da pressão de entrada. Esses resultados também seguem o comportamento dos resultados encontrados por Carreiro (2009), onde o autor analisa um sistema de evaporação com 32.186,7589KW de exergia de entrada, obtendo uma eficiência exergética 1,2\% menor do que em um mesmo sistema de evaporação com 29.031,1942KW de exergia de entrada.

Os resultados do modelo mostraram um aumento da eficiência exergética de 6,60\% e teor de sólidos de 4,34\% quando a pressão de entrada foi diminuída de $420 \mathrm{kPa}$ para $380 \mathrm{kPa}$, acompanhada de uma diminuição de exergia destruída de 7,49\%. Com a pressão diminuída de $420 \mathrm{kPa}$ para $400 \mathrm{kPa}$ (Pressão de referência), há um aumento de 3,66\% na eficiência exergética, de $2,31 \%$ no teor de sólidos e $0,33 \%$ na exergia destruída.

De acordo com Passini (2017), um dos motivos que causa essa diminuição da eficiência exergética, quando se aumenta a pressão de entrada, é o fato de haver uma maior taxa de incrustações nas paredes internas dos evaporadores, não calculado nesse trabalho, acarretando uma diminuição na área de troca térmica e dificultando o bombeamento do licor. Entre os valores de entrada estudados, o de $380 \mathrm{kPa}$ se destacou por possuir melhores valores de eficiência exergética, de teor de sólidos e destruição de exergia. O gráfico a seguir ilustra a taxa de crescimento de teor de sólidos do licor negro.

Figura 2 - Percentual teor de sólidos, exergia destruída e eficiência de segunda lei.

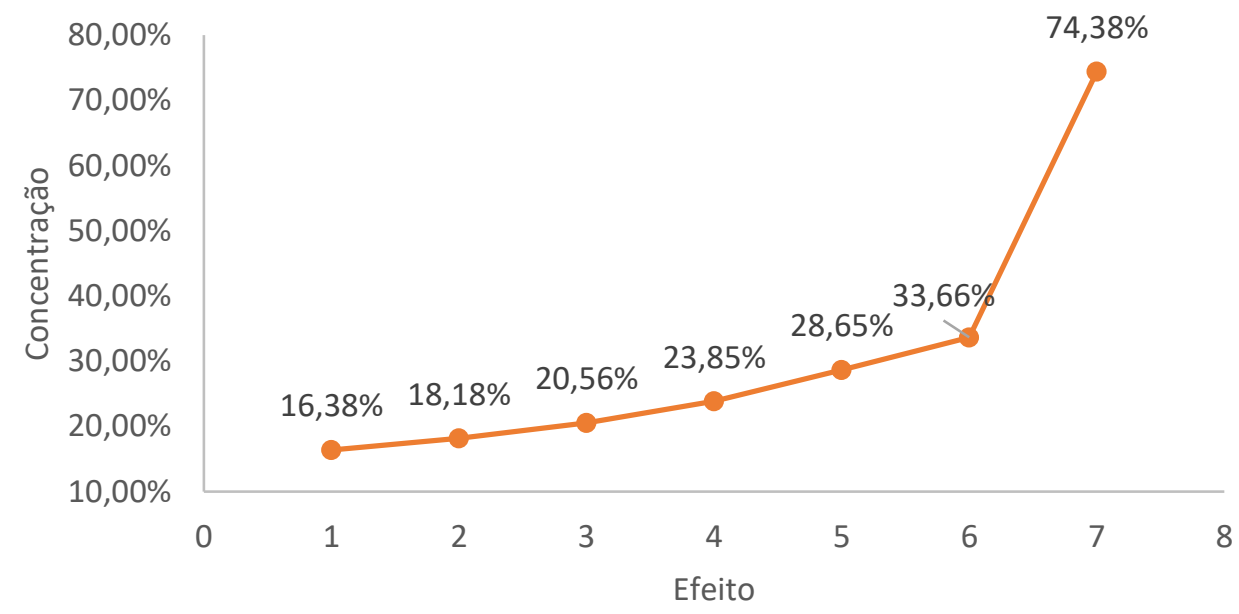

Fonte: Elaboração própria (2021).

Quanto à exergia destruída e à eficiência, percebe-se que os primeiros 6 evaporadores apresentam resultados parecidos, variando em margens de valores próximas. No posicionamento de Guerato (2012), o último evaporador apresenta uma taxa de destruição maior, por se tratar de um evaporador em série, composto por dois evaporadores e pelo fato de haver mais incrustações pela elevada concentração do licor. Dessa forma, vê-se que o modelo matemático também convergiu para os resultados encontrados pelo autor citado, como são mostrados na ilustração abaixo. 
Figura 3 - Taxas de destruições de exergia e eficiências de segunda lei.

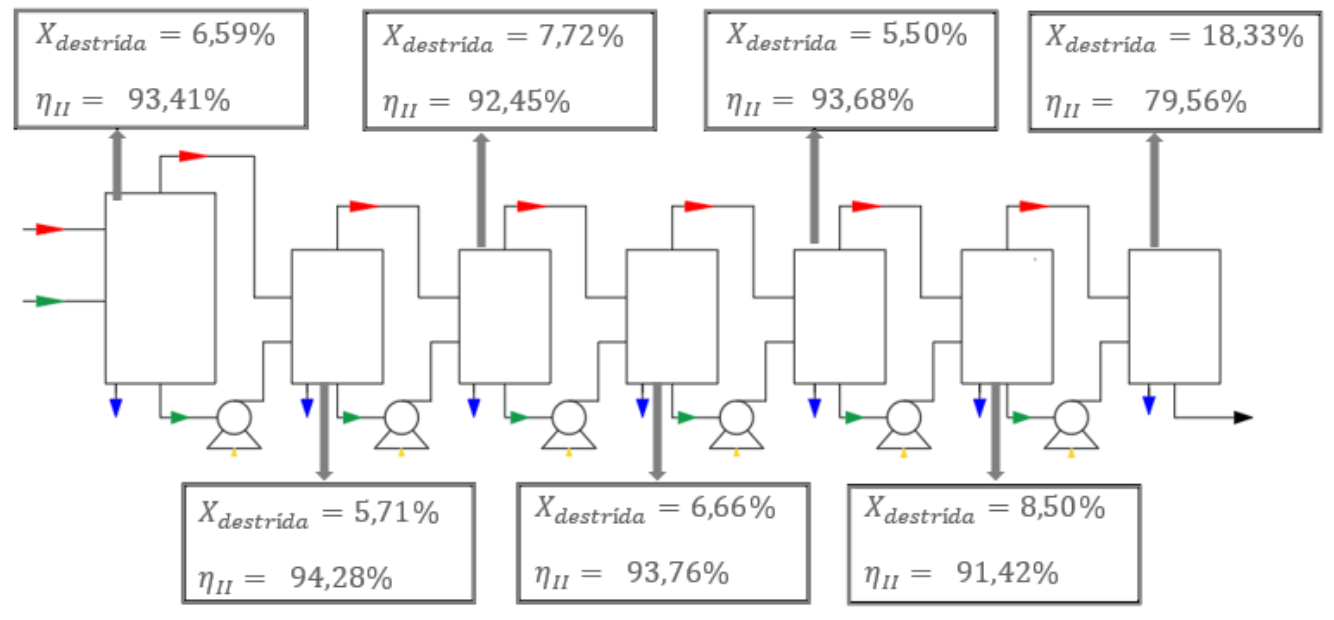

Fonte: Elaboração própria (2021).

Segundo Ponte, Barbosa e Rufino (2010), a existência de incrustações, resultante da queima incompleta de material, diminui a transmissão do calor do combustível para o processo na caldeira e, consequentemente, sua eficiência. Conforme é dito por Silva (2016), quanto maior a concentração de sólidos de licor negro, maior a temperatura de ebulição, o que afeta diretamente na eficiência dos evaporadores. A partir disso, podemos perceber que conforme o processo vai avançando através dos evaporadores, descrito na Figura 3, os valores de destruição de exergia tendem a aumentar à medida que a eficiência diminui através dos estágios com licor.

\section{Considerações Finais}

A melhoria na eficiência exergética de equipamentos termodinâmicos nas indústrias é fundamental para o aprimoramento do processo produtivo e redução de custos, com o resultado de tornar as empresas mais competitivas no âmbito econômico. Com isso, este artigo analisou, por meio de uma modelagem matemática e fenomenológica, dentre as condições de entrada, a que trouxesse os melhores valores de concentração de sólidos e de eficiência exergética em um sistema de evaporação de múltiplos efeito de uma empresa de papel e celulose brasileira. O modelo matemático foi composto por equações de balanço de massa, energia, propriedades físicas e termodinâmicas aplicadas em todos os efeitos do sistema de evaporação estudado, as pressões de entrada analisadas foram adotadas a partir dos valores encontrados nos principais trabalhos usados como referência para esta pesquisa.

Visto ao exposto, a modelagem matemática realizada com balanços de massa, energia, propriedades físicas e termodinâmicas, foi capaz de predizer os resultados encontrados por Carreiro (2009) com boa precisão, sendo o maior desvio de $0,20 \%$. A condição de entrada que deteve a melhor eficiência exergética foi a de $380 \mathrm{kPa}$ com o valor de 45,86\% e um teor de sólidos de 74,38\%. Observou-se uma eficiência de Segunda Lei do primeiro ao sexto efeito superior ou igual a 91,42\%. Entretanto, o sétimo efeito apresentou uma baixa eficiência de Segunda Lei de 79,56\%, por criar mais incrustações nas paredes internas e possuir um evaporador em paralelo. Na melhor condição de entrada encontrada, $380 \mathrm{kPa}$, houve uma economia de fluxo de vapor vivo de $20 \mathrm{kPa}$ em relação ao trabalho de referência, com um aumento de eficiência de $2,31 \%$ e de teor de sólidos de 4,34\% para concentrar 15,094 kg/s de licor negro.

Este estudo foi capaz de encontrar condições de funcionamento de um sistema de evaporadores de múltiplos efeitos com melhores valores de eficiência, concentração no teor de sólidos de licor negro e economia de vapor a partir da criação de uma nova metodologia de análise criada nesta pesquisa. Com isso, a melhor condição de vapor de entrada, no valor $380 \mathrm{kPa}$, 
trará uma economia de vapor que reduzirá custos de produção na empresa e redução na emissão de gases poluentes devido a redução de combustão nas caldeiras para gerar uma menor quantidade de vapor necessária. Foram encontradas algumas limitações durante a execução do trabalho, como a ausência dos valores de redução de pressão entre os efeitos do trabalho usado como referência para validação do modelo matemático e de alguns dados que possivelmente são tidos como segredos da indústria.

Com a validação dos resultados encontrados nesse trabalho, viu-se que a modelagem matemática realizada no presente trabalho apresentou bons resultados. Como sugestão de trabalhos futuros, propõe-se a criação de uma interface interativa para realização de cálculos de concentração de licor negro e eficiência exergética de sistemas de evaporadores de múltiplos efeitos com configurações diferentes da que está presente neste trabalho. Ademais, sugere-se, também, a criação/implementação de uma metodologia capaz de mensurar o nível de incrustação presente em cada efeito do sistema de evaporadores de múltiplos efeitos aplicados em indústrias de papel e celulose.

\section{Agradecimentos}

Os autores agradecem o apoio do Instituto Federal do Piauí (Campus Teresina Central) pelo financiamento do desenvolvimento desta pesquisa por meio concessão de bolsas do Programa Institucional de Bolsas de Iniciação Científica PIBIC (Edital N 7843 de 28 de setembro de 2020).

\section{Referências}

Andreuccetti, M. T. (2019). Estudo experimental e teórico da solubilidade de sais de sódio em licor negro no processo de evaporação. Tese (doutorado) Universidade Estadual de Campinas.

Batista, T. S. (2018). A indústria de papel e celulose no brasil: produtividade, competitividade, meio ambiente e mercado consumidor. Monografia (Curso de Engenharia Química), Universidade Federal de Uberlândia.

Bhargava, R., Khanam, S., Mohanty, B., \& Ray, A. K. (2008). Simulation of flat falling film evaporator system for concentration of black liquor. Computers \& Chemical Engineering, 32(12), 3213-3223.

BNDES, Banco Nacional de Desenvolvimento Econômico e Social. (2012). BNDES 60 anos: perspectivas setoriais. BNDES.

Campos, S. G. (2009). Modelagem matemática e análise do coeficiente global de transferência de calor para o processo de concentração do licor negro de eucalipto em sistema de evaporadores de múltiplo efeito. Dissertação de mestrado (Programa de Pós-Graduação em Engenharia), Centro Universitário do Leste de Minas Gerais, Coronel Fabriciano - MG, Brasil.

Cao, C., He, Y., Chen, J., Cao, W., \& Jin, H. (2018). Evaluation of effect of evaporation on supercritical water gasification of black liquor by energy and exergy analysis. International Journal of Hydrogen Energy, 43(30), 13788-13797.

Carreiro, M. R. M. (2009). Análise exergética e ambiental do processamento do licor negro em fabricas de celulose e papel. Dissertação (Mestrado) Universidade Federal de Itajubá.

Cengel, Y. A., Boles, M. A., \& Kanoglu, M. (2011). Thermodynamics: an engineering approach (Vol. 5, p. 445). McGraw-Hill.

Diel, C. L. (2013). Modelagem matemática do processo de evaporação do licor negro proveniente do sistema de lavagem da polpação "Kraft”. Dissertação (Mestrado), Universidade Estadual do Oeste do Paraná, Toledo - PR, Brasil.

Ferreira, D. J. O. (2013). Modelagem de caldeira de recuperação química Kraft. Tese (Doutorado em Engenharia Química), Universidade de São Paulo.

Gallo, Valdir. (1998). Avaliação da Entropia pela Segunda Lei da Termodinâmica, Notas de Aula, Universidade Estadual de Campinas.

Guerato, G. (2012). Otimização dos evaporadores tipo falling film de uma planta de evaporação do licor negro proveniente do processo de digestão da madeira - Kraft, através da modificação do processo de lavagem visando o aumento da capacidade da unidade. Monografia (Graduação em Engenharia Química), Universidade de São Paulo, Lorena - SP, Brasil.

Ibá, Indústria Brasileira de Árvores. (2018). Exportações do setor crescem 10,7\% em 2018. Brasil.

Kotas T. J. (1995). The Exergy Method of Thermal Plant Analysis, reprint ed. Krieger, Elsevier, Malabar.

Marcelino, Thamires Ohana Araújo de Carvalho. (2019). Modelagem e Simulação da etapa de Caustificação do Processo Kraft de Extração de Celulose. Dissertação (Pós-Graduação em Engenharia Química), Universidade Federal de Campina Grande.

Mendes, A. S. (2021). Modelagem de um sistema de cogeração com motor a diesel integrado a unidade de dessalinização multi-efeito. Monografia (Curso de Engenharia Mecânica), Universidade Federal do Rio Grande do Norte, Natal-RN, Brasil. 
Research, Society and Development, v. 10, n. 15 e221101522835, 2021

(CC BY 4.0) | ISSN 2525-3409 | DOI: http://dx.doi.org/10.33448/rsd-v10i15.22835

Moreira, J. (2018). Análise exergética do sistema de evaporadores de múltiplo efeito da etapa de recuperação da indústria Kraft de obtenção de celulose. Dissertação (Pós-Graduação em Engenharia Química), Universidade Federal de Minas Gerais, Belo Horizonte - MG, Brasil.

Olsson, M. R., \& Berntsson, T. (2007). A tool for simulating energy-efficient evaporation using excess heat in kraft pulp mills. In Proceedings of the 2007 Engineering, Pulping \& Environmental Conference, Oct 21-24, 2007, Jacksonville, FL, USA.

Passini, R. J. (2017). Análise exergética de um sistema de recuperação química de uma fábrica de papel e celulose. Dissertação (Mestrado) - Curso de Engenharia Mecânica, Programa de Pós-graduação em Engenharia Mecânica, Universidade Federal de Itajubá, Itajubá (MG), Brasil.

Ponte, R. S. T; Barbosa, M. A. P. \& Rufino, M. G. (2010). Eficiência energética em caldeiras. Congresso Brasileiro de Planejamento Energético. Energia 2030: Desafios para uma nova Matriz Energética. São Paulo - SP, Brasil.

Seleghim, P. (2018). Termodinâmica: T8 Exergia 2/3 Sistemas de evaporação multiestágios - Evaporadores de caldo. https://www.youtube.com/watch?v=s1sTfWZL_cU\&list=PLmho8Rcnd60fFdZ1oItM7_lt43L4Po8Fi\&index=14

Silva, C. A. F. et al. (2016). A indústria de celulose e papel no brasil: A inovação é o que faz a diferença entre um líder e um seguidor. Guia ABTCP.

Silva, R. M. (2016). Estudo de aumento de eficiência e produção de uma caldeira de recuperação química. Monografia (Curso de Engenharia Mecânica), Universidade Estadual Paulista, Guaratinguetá-SP, Brasil.

Stape, G. J. (2017). Simulação de unidade de recuperação química do processo de polpação kraft visando a obtenção de metanol celulósico. Dissertação (PósGraduação em Engenharia Química), Universidade de São Paulo. 